\title{
Costs of physical health care for self-injuring patients
}

\author{
Mark Swinton and Sarah Smith
}

\begin{abstract}
Self-injury is a major clinical problem on the women's wards at Ashworth Hospltal. Ashworth Hosplital is one of three special hospltals within England and Wales that provides assessment, treatment and rehabilitation for those legally detained patients under the Mental Health Act (1983) who suffer from a mental disorder and require conditions of special security because of their dangerousness. There is extensive literature on the psychological aspects of self-injury. However, since patients with self-injury present with damage to the body, it is surprising that there is little information on the physical health care and associated resource costs of this behaviour.
\end{abstract}

This study estimates the physical health care costs that result from an episode of self-injury. Patients' status as long-stay in-patients at a psychiatric hospital was not changed by individual episodes of self-injury and thus psychiatric healthcare costs have not been estimated.

This study describes the costs of providing physical health care to self-injuring female patients at Ashworth Hospital. Over three years the numbers of women patients fell from 73 (April 1993) to 65 (April 1994) to 60 (April 1995). For the purpose of this study, self-injury was regarded as deliberate damage to one's own body irrespective of motivation and which required some form of intervention. The study included the acts of overdosing of drugs and of swallowing foreign bodies, because although they do not always cause actual bodily damage, intervention is required because of the possibility of damage. By definition the study excluded minor acts of self-injury that do not require physical intervention (e.g. head-banging, eye-rubbing).

There are high levels of psychiatric nursing staff on the wards where self-injuring patients are found, with the intention of preventing serious self-injury. This study is limited to measuring the cost of physical health care and the cost of this psychiatric care has not been included.

\section{Physical health care available to in-patients}

The primary physical care of the patients at Ashworth Hospital is undertaken by a local general practice which provides a surgery each weekday morning and a 24-hour on-call service for medical emergencies. GPs are paid a fixed annual sum regardless of the number of patients they see, and receive a fixed extra payment to cover out-of-hours visits.

Consultants in accident and emergency (A\&E) medicine from the local general hospital come in to the hospital to provide treatment or advice at the request of nursing staff. The medical centre at Ashworth Hospital provides an X-ray machine operated by two part-time radiographers and overseen by a part-time consultant radiologist. Patients can be transferred to one of the local general hospitals when necessary.

\section{Types of self-injury requiring intervention}

(1) Cutting of the skin requiring sutures. The practice at this hospital is for the ward nurses to call one of two consultants from the local A\&E department who come to the hospital and provide suturing as required.

(2) Swallowing. A number of patients frequently swallow small objects. These typically cause no symptoms and pass naturally. On occasion patients swallow larger objects (aerials and the metal band of headphones) that are too big to pass through the gastrointestinal tract. These must be removed by endoscopy or through invasive surgical procedures. In addition, patients insert foreign bodies into existing or newly prepared wounds or into an orifice.

The great majority of treatment interventions follow these two types of self-injury. In addition, a number of other methods of self-injury occur less frequently.

(3) Overdose of medication. Medication is handed out by nursing staff. However, some patients secrete medication or obtain it from other patients, and subsequently overdose. These patients are taken to a local casualty department for appropriate treatment. 
(4) Ligature tying. Patients make ligatures out of lengths of material and tie it tightly around their necks (usually without suspension) often leading to some reduction of consciousness. On most occasions these events take place in circumstances where nurses can intervene quickly and there is no need for any physical treatment. However, on occasions this does not happen and patients need to be taken to the local A\&E department for further treatment. In addition. patients attend the department for:

(a) suturing if the A\&E consultant is on leave or otherwise unavailable;

(b) X-ray if it is thought clinically unsafe for the patient to wait until the $\mathrm{X}$-ray facility is next available within the Ashworth Medical Centre;

(c) specialist opinion: the A\&E consultants refer patients to the on-call teams in the general hospital to decide if further treatment is necessary.

\section{Method: estimate of costs}

(1) Emergency consultant call-outs. The call-outs of $A \& E$ consultants to the wards were recorded by log books filled in at the time of each episode. Data were available for three years up to April 1995. The cost of this service was $\$ 97.00$ per episode in 1994/95 and slightly less in earlier years.

(2) Hospital admission. When an in-patient from Ashworth Hospital is admitted to local hospitals, the cost of medical and surgical treatment is billed as an extra-contractural referral (ECR) back to the health authority from which the patient originated. ECR costs for all Ashworth Hospital in-patients were available for the two years from April 1993 to April 1995. Nursing staff from Ashworth Hospital escort patients whenever they are out of the hospital. As a matter of policy all female patients in hospital are escorted by a minimum of two nursing staff, usually one qualified nurse (Grade $D$ to $F$ ) and one nursing assistant (Grade A or B). Staff are recruited on an overtime basis for this work, often at short notice over nights and weekends. The average cost of escorting nursing staff was estimated at $£ 30.00$ per hour throughout the period of hospital stay. We also calculated the total period the patients spent in hospital.

(3) Emergency visits to the local A\&E department. Data on emergency transfers to A\&E were retrieved. Included in this group were overnight stays on the emergency ward. The treatment cost for attendance at the $A \& E$ department is borne by the local hospital's A\&E budget, as all patients are registered with the local general practice and treated as local residents for this purpose. The average cost for an $A \& E$ attendance (as supplied by the Finance Department of the relevant hospital) was $\$ 43.00$ per visit. Nursing escort costs were charged on the same basis as that for in-patient stays.

(4) X-ray costs. The Ashworth Hospital service was available between 9 am and $3 \mathrm{pm}$. Outside this time X-ray work was carried out at the local $A \& E$ department. It was established that over a one-year period, $11 \%$ of all X-rays were related to self-injury, primarily to identify the location of foreign bodies. Eleven per cent of the total budget of the Ashworth Hospital X-ray department (£4242) was identified as a cost of self-injury.

(5) Transport costs. As well as taking patients to and from general hospital, all escorting staff had to be transported between the outside hospital and Ashworth at the start and end of shifts.

(6) General practitioners. GPs were not involved in the immediate management of self-injury. However they were consulted by women patients as a result of some of the physical consequences. Typically this was because of pain following previous self-injury or in situ foreign bodies. However, these patients had a high consultation rate due to other physical complaints and it was not possible to establish which consultations were due to self-injury alone. No estimate has been made of the costs of providing a GP service in connection with self-injury.

\section{Findings}

\section{Emergency consultant call-out}

Table 1 gives the number of call-outs, estimated cost and the number of patients involved. In 1994/95 most call-outs followed episodes of cutting (271 calls: $61.3 \%$ ) and insertion of foreign bodies ( 132 calls: $29.9 \%$ ). In all three years, these two causes accounted for over $90 \%$ of call-outs.

Over each 12-month period the actual number of patients needing a call-out fell but remained in a stable proportion to the number of in-patients (1992/3: 49\%; 1993/4: 52\%; 1994/5: 50\%). The distribution of call-outs to patients is highly skewed with a few patients accounting for most calls. Each year five patients have accounted for around half ( $49 \%, 46 \%$ and $55 \%)$ of all call-outs.

The main change over time was the number of call-outs for each patient having one call-out. The median more than doubled over this period. The rise in call-outs between 1992/93 and $1993 / 94$ is statistically significant (Wilcoxon matched-pairs signed-ranks test: $z=-2.01$, $P=0.044)$. The rise between $1993 / 94$ and 1994 / 95 is not significant.

\section{Hospital admission}

There were 53 admissions during 1993/94 and 37 during 1994/95. The ECR costs were $£ 48000$ 
ORIGINAL PAPERS

Table 1. Emergency consultant call-outs

\begin{tabular}{lccc}
\hline & $1992 / 93$ & $1993 / 94$ & $1994 / 95$ \\
\hline Number of calls & 231 & 366 & 445 \\
Cost $(\varepsilon)$ & 21216 & 34477 & 42874 \\
Number of patients with at least one call & 36 & 34 & 30 \\
Median calls (for patients with at least one call) & 3.0 & 4.5 & 8.5 \\
\hline
\end{tabular}

and $\$ 47824$ respectively. The estimated escort costs were $£ 109170$ and $£ 111135$ respectively. Procedures for which the women attended general hospital included endoscopy, abdominal or thoracic surgery to remove swallowed foreign bodies, or observation following self-injury.

Sixteen patients had an admission. Two patients (described here as patient $A$ and patient B) had 34 and 28 admissions respectively, accounting for $66 \%$ of admissions. One patient had nine admissions and one patient seven admissions. The other 12 patients had one or two admissions.

There were 15 major surgical operations (nine laparotomies and six thoracotomies). Patients $A$ and $B$ had seven major operations each. These were performed to remove swallowed foreign bodies. One other patient had a single major operation; this was also to remove foreign bodies. There were 26 episodes of non-operative removal of swallowed bodies (endoscopy or sigmoidoscopy): patients $A$ and $B$ had ten each. In 42 admissions there was no specific procedure: this included admission for observation and patients taking their own discharge. The 13 other admissions covered a variety of procedures.

Altogether there were 301.4 hospital in-patient days over the two years. Patient $A$ had 110.2 inpatient days and patient $B$ had 91.9 in-patient days. These two patients accounted for $67 \%$ of all in-patient days. During this two-year period, patient $A$ was in a medical or surgical ward for $15 \%$ of the time and patient B for $13 \%$ of the time.

In $1994 / 95$ there were 68 visits to the local A\&E department. The attendance cost was $£ 3124$ and the escort cost was $\$ 12510$. Costs over the previous two years were of similar magnitude.

Table 2 gives an estimate of overall costs for 1994/95.

\section{Comment}

Special hospitals are not unique in having patients who self-injure. All psychiatric services have such patients. Special hospitals are unusual, however, in having large numbers of self-injuring, personality disordered in-patients remaining in hospital for long periods of time.
Table 2. Total estimated cost for the physical treatment of self-injury in 1994/95

\begin{tabular}{lr}
\hline Area of activity & Cost (\$) \\
\hline Consultant call-out & 42874 \\
A\&E treatment & 3124 \\
A\&E escort & 12510 \\
In-patient treatment & 47824 \\
In-patient escort & 111135 \\
Transport & 5553 \\
X-ray & 4242 \\
Total health service cost & 227162 \\
\hline
\end{tabular}

The responsibility for providing physical health care following self-injury is widely dispersed. The overall cost to health services following selfinjury by patients at this hospital is approaching a quarter of a million pounds a year. Ashworth Hospital alone is spending over $£ 170000$ per year. It is important to recognise that this money is spent in an essentially non-productive way in that it merely returns patients to the state they were in before they self-injured. Money spent in this way is not available for providing psychiatric treatment services.

The study has not covered all possible costs. As noted above, the costs of the GP service could not be measured. The costs of providing psychiatric health care to self-injuring patients were not measured. In addition, indirect costs (the loss of productivity due to episodes of illness) were not measured. Although there are a number of areas of expenditure, the major cost is that of providing in-patient hospital care, particularly that which follows major surgical operations. Two patients alone account for the great majority of this work. These were two patients who repeatedly swallowed large objects. It follows that specific attention to these two patients, and considering alternative strategies for them, could produce significant resource savings. These two patients spent long periods in surgical wards with consequent disruption of attempts at sustained psychiatric treatment.

Clinical practice changed once the data became available. It was recognised that both 
patients were typically swallowing aerials and headphones. During early 1995 the ward where these two patients were located removed all headphones with metal from patients, replacing them with wire headphones which cannot be used in the same way. The two patients were not allowed to have any equipment with aerials. Other patients were allowed to have equipment with aerials as long as they were kept locked within their room. Patient B did not swallow over the next year.

Patient A was put under special obervation (a nurse was with her throughout her waking hours) over a 140-day period. This was due to increasing concern over her ability to tolerate continuing major surgical procedure. She did not swallow during this time. Based on her pattern over the previous two years it can be predicted that she would otherwise have spent approximately 21 days as an in-patient on a surgical ward having undergone one or two major surgical operations. The gain from the special observation policy was a cessation of swallowing with a reduction in risk to physical health. In addition, the patient was able to remain in the psychiatric hospital and was able to see a psychologist regularly, something which had not been possible in the past. On the other hand. the patient found the nursing approach very intrusive (and pressed for its discontinuation), and it was difficult for nurses to remain enthusiastic for long periods. After almost five months the policy had to be discontinued and this was followed by a return to the previous pattern of swallowing.

In conclusion, dealing with physical care after self-injury imposes a high cost on this service. Most of this cost can be attributed to a few patients and extra attention needs to be paid to this group.

"Mark Swinton, Senior Lecturer in Forensic Psychiatry, Liverpool University and Sarah Smith, Team Leader, Ashworth Hospital, Liverpool

*Correspondence

\title{
Books from Gaskell
}

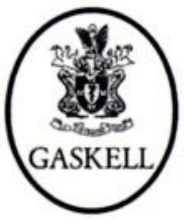

\section{Mental Disorders in Cbina}

\author{
English Version Editors: John E. Cooper \& Norman Sartorius
}

This is the first available account in English of the results of the 1982 Chinese Epidemiological Survey of Mental Disorder. It is the only nationwide Chinese study to involve modern methods of case assessment and is uniquely large and interesting; 12 centres in different parts of China studied a rural and an urban sample of 500 households each, giving a total sample of 51982 persons. The use of the Present State Examination in the survey allows the symptomatic basis of diagnoses, such as neurasthenia, to be examined. The results indicate that the prevalence of schizophrenia is similar to that found recently in other countries, but that the prevalence of other mental disorders may be lower. Readers may also be interested to purchase Mental Health Care in China, published in 1995, price $£ 12.50$.

$$
\text { - Price } f 15.00 \bullet \text { I28pp. • I996• ISBN } 0 \text { 90224I } 93 \text { I }
$$

Available from good bookshops and from the Publications Department, Royal College of Psychiatrists, I 7 Belgrave Square, London SWIX 8PG (Tel. 0171 -235 235I, extension 146) 